\title{
The Variation Pattern and the Distribution Comparative Analysis of Bacterial Pathogenic Spectrum of Children from Different Age Groups and Adults
}

Shifu Wang

Yang Li

Weiping Zhou

Renzhe Li

Yanping Chen

Zhiwei Liu

Dequan Zhu

Yanhua Li

Yilei Li

Haiying Chen

Sijin Man

Jiliang Wang

Jin Li

Qingling Li

Changwu Zhang

Maoli Yi

Tao Chen

Mingju Hao

Fen Su

Yuanqi Zhu

Bin Zhou

Xiaolin Luan

Liming Shi

Mingyan Sun

Xiaoying Li

Xu Jing

Haizhu Liu

Zhiyong Zhang

Hongyang Zhao

Qi Ran

Bin Ji

Bo Li 
Guanghui Zhang

Xu Zheng

Chengjie Guo

Xuejiao Leng

$\mathrm{He} Y \mathrm{i}$

Jiewei Wang

Guijun Xu

Liming Shi

Hong Zhang

Jing Li

Yunkui Zhan

Zhongtao Gai ( $\nabla$ gaizhongtao@hotmail.com )

Research article

Keywords: Children; Adults; Age; Season; Gender; Variation pattern; Pathogenic spectrum

Posted Date: June 13th, 2019

DOI: https://doi.org/10.21203/rs.2.10256/v1

License: (9) This work is licensed under a Creative Commons Attribution 4.0 International License.

Read Full License 


\section{Abstract}

Background To obtain the pathogenic spectrum of infectious diseases of different development phases of children from different regions of Shandong Province, we collected the data of pathogens from 40 hospitals in 17 cities of Shandong Province, and aimed at promoting the management and reasonable application of children's antibacterial agents. Methods We collected the bacterial drug resistance monitoring information from 26,778 strains isolated from children from 40 hospitals in 17 cities of Shandong Province (including 35 Grade 3A tertiary hospitals and 5 secondary hospitals) in 2017, and analyzed the variation pattern and the comparative distribution features of the pathogenic spectrum changing with age, season and gender. Results Pathogenic spectrum changes along with children's age. For example, Streptococcus pneumonia and Haemophilus influenza show a significant feature of normal distribution as the age increases. The distribution of pathogenic spectrum is significantly affected by season change. Infections reveal inequality between the sexes. Conclusion We first established a provincial-level pediatric antimicrobial surveillance system (Shandong Province Pediatric Antimicrobial Resistance Surveillance System, SPARSS) in China. Supported by the analysis of big data for the first time, we confirmed that the pathogenic spectrums for children from different development stages and seasons are completely different. Besides, we also found the evidence for facts that men in general live shorter lives than women, and boys are more easily to be infected compared with girls. In order to promote reasonable and standard application of antimicrobial drugs on children, clinical doctors should choose antibiotics according to the results of the variation pattern and the distribution comparative analysis of pathogenic spectrum for children from different age groups concluded by our study.

\section{Background}

Antibiotic resistance has now become a serious global public health crisis [1]. Although the clinical application of antibacterial drugs management method has been effectively implemented in China since August 1, 2012, until now there is no national or provincial report of bacterial drug resistance monitoring data in China especially for such special group as children. There is also no big data statistical study on children's pathogenic spectrum or the variation pattern of the pathogenic spectrum of children from different age groups in China. Without pathogenic spectrums of children from different age groups, clinical doctors find it hard to use the antibiotics reasonably as one cannot make bricks without straw. Therefore, reasonable use of antibiotics is essentially groundless [2].

To study the variation pattern of pathogenic spectrum for children from different age groups and conduct the distribution comparative analysis of the pathogenic spectrum between children and adults from multiple laboratories in Shandong Province, the SPARSS was established in 2017, including 40 laboratories (from 35 Grade $3 \mathrm{~A}$ tertiary hospitals and 5 secondary hospitals) in 17 cities of Shandong Province. The data, collected from microbiology laboratories in a uniform format, were put into a central database using WHONET 5.6 software [3]. In this analysis, we established the first provincial-level surveillance network for children bacterial resistance in China. These data first provided a comprehensive 
picture of pathogenic spectrums in different regions of Shandong Province and different development phases of children.

\section{Methods}

Materials

Source of Strains

All aerobic bacteria (excluding anaerobic bacteria and fungi) collected from outpatients and inpatients in 40 hospitals were obtained from the SPARSS in 2017. Most of the hospitals included are the largest general hospital and maternal \& child care hospital in each prefecture-level city of Shandong Province. They can represent the data of 17 prefecture-level cities (about 100 million population). 87,133 isolates from adults and 26,778 isolates from children were collected from 40 hospitals, including 35 Grade $3 \mathrm{~A}$ tertiary hospitals and 5 secondary hospitals. The bacterium specimens cover the blood, bronchoalveolar lavage fluid, sputum, pus specimens, stool, cerebrospinal fluid, and various kinds of secretion of patients ranging from new-born babies to 18-year-olds.

The monitoring duration was from January 1, 2017 to December 31, 2017. The strain data for statistics and analyses strictly followed these criteria: Only the beta hemolytic streptococcus of the throat samples was monitored. Among lower respiratory tract specimens, only the pathogenic bacteria and the growing pathogenic bacteria were monitored. Only intestinal pathogenic bacteria of the fecal specimens were monitored. Only urine specimen isolates whose colony count were equal to or greater than 104 colony forming units $(\mathrm{cfu}) / \mathrm{ml}$, and whose bacterial species were equal to or less than 2 were monitored.

Exclusion criteria: Repetitive strains and the normal strains of the skin isolated from the sterile area were excluded. For Coagulase-negative Staphylococcus and Viridians Streptococci, only isolates collected from blood and cerebrospinal fluid were included in the analysis.

Bacterial Strains, Culture Media and Species Identification

In order to avoid repetitive counts, for every patient, only one isolate from the same species was included based on the personal identifying code and one's hospital. Species identification of the isolates was performed by standard biochemical methods using the Microsan Walkaway (Siemens, Germany) and Vitek 2 automated system (bioMérieux, France).

Antimicrobial Susceptibility Testing

The antibiotic susceptibility of clinical isolates was determined using the minimum inhibitory concentration and disk diffusion method according to the Clinical and Laboratory Standards Institute criteria ( http://ncipd. org/control/images/NCIPD_docs/CLSI_M100-S28.pdf).

Reference Strains 
Staphylococcus aureus ATCC (American Type Culture Collection) 25923, Enterococcus faecalis ATCC 29212, Escherichia coli ATCC 25922 and Pseudomonas aeruginosa ATCC 27853 were included to ensure reproducibility of the antibiotic susceptibility testing procedure.

Methods

The Principle of Age Grouping

According to the characteristics of children's physical development, diet and external contact with pathogens, the subjects were divided into the newborns group ( $0 \sim 28$ days), babies group ( 29 days $\sim 6$ months), the young children group (7 months $\sim 3$ years), preschoolers group ( $4 \sim 6$ years), teenagers group (7 18 years), and adults group (above the age of 18).

Statistical Analysis

The pathogenic spectrum distribution analysis of children and adults was carried out by using the WHONET5.6 statistical analysis software for comparative analysis.

\section{Discussion}

We have established the first provincial-level pediatric antimicrobial surveillance system (SPARSS) in China to obtain the variation pattern of pathogenic spectrums in children from different development stages, conduct the distribution comparative analysis of pathogenic spectrums between children and adults, detect various children's infection of pathogenic microorganisms to various antibiotics sensitivity, report children bacterial drug resistance monitoring data in Shandong Province, improve relevant medical and health institutions of bacterial infection and treatment ability, and promote management and reasonable application of children's antibacterial agents. The SPARSS network covered 17 prefecturelevel cities in Shandong Province, including 35 tertiary hospitals and 5 secondary hospitals. The hospitals are uniformly distributed with significant regional representativeness (see Figure 1), so the monitoring data can basically represent the pathogenic spectrum of children and adults in Shandong Province. The pathogenic spectrum of adults in Shandong Province is basically consistent with the result of CHINET surveillance net in China that focuses on the adult antimicrobial resistance surveillance. So our data should be related thereto and can represent the surveillance data of the whole China because Shandong is in the middle part of China. Hence, our conclusion for children pathogenic spectrum and the variation pattern is not only useful for China, but also for doctors in other countries and regions of the same latitude.

The percentage comparison of main pathogens between children (26,762 strains) and adults $(87,133$ strains) confirmed that children and adults are significantly different in pathogenic spectrum. Staphylococcus aureus, Streptococcus pneumoniae, Haemophilus influenzae and Moraxella catarrhalis bacteria are closely related to children's immune function and anatomical structure of children's characteristics of strains [9]. The related papers have reported that the separation rate is much higher for 
children than the adults [10]. Children have their unique characteristics of physiological development and pathogenic spectrum. This may affect children's immune function which is not fully mature, and can easily acquire acute upper respiratory tract infection. Respiratory pathogens in nasopharynx is closely related to factors such as the number of carrying rate and engraftment $[9,10]$. We confirmed, according to the results from the big data analyses, that the bacterial infection of pathogenic spectrum of children is also completely different from that of adults [10].

By analyzing the change trend of the percentage of main pathogens in children of different age groups, we found that the strain of Staphylococcus aureus in all age groups basically occupies the first or the second place (see Table 1). Only in children's group aged from 7 months old to 3 years old and group aged from 7 years old to 18 years old, the percentages of Streptococcus pneumonia and E.coli are higher than those of the Staphylococcus aureus. E. coli gradually goes from the third place to the first place in group aged from 7 years old to 18 years old, which is similar to adult pathogenic spectrum.

Streptococcus pneumoniae and Haemophilus influenzae show significant characteristics of normal distribution as the age goes up. The number of isolated strains reaches a minor peak in the group aged from 7 months old to 3 years old. This indicated that, according to their distribution characteristics, we should pay more attention to this age group, and doctors should attach great importance to the detection of Streptococcus pneumoniae and Haemophilus influenzae strains. According to our finding, we suggest that doctors combine it with actual circumstances when they choose antibiotics according to the state of illness and the part of infection. The treatment they selected for children aged from 7 months old to 3 years old should also be based on factors, such as medical history and season, especially covering the two pathogenic bacteria mentioned above.

By analyzing the monthly variation change trend of main pathogens in children, the monthly number variation of Haemophilus influenza is similar to that of the Streptococcus pneumoniae. As we have known Streptococcus pneumoniae and Haemophilus influenza are main upper respiratory pathogens in children. They increased from September to next March almost at the same time. The change trend may be related to the flu season. As we all know seasonal influenza is a large burden during each wintertime $[6,11]$. The increasing evidences show that influenza virus interacts with other pathogens colonizing or infecting the human respiratory tract, especially children [4]. The main pathogens may be critical to the decision of the rational use of antibiotics targeted the secondary bacterial infection [6]. The effect of the interval between influenza infection and bacterial acquisition reportedly affects the risk of bacterial invasion $[12,13]$. Our result confirmed that pathogens and influenza virus are like a band of robbers. Firstly the influenza virus opened the doors of upper respiratory endothelial barrier in children, and then the upper respiratory main pathogens, Streptococcus pneumoniae and Haemophilus influenza, easily pass through the upper respiratory endothelial barrier, and cause the bacterial infections of upper respiratory tract, bronchitis and pneumonia in children.

It have been reported that sexual reproduction can play a critical role in the origin of the outbreak of strains' infections [14]. The immune system's responses to infection of men and women are very different. Some publications, which have confirmed that immune responses of women and men are 
different, explained that women might have evolved a particularly strong and fast primary immune response in order to protect the developing fetuses and newborn babies. This might explain the reason why more women tend to develop autoimmune diseases such as multiple sclerosis and lupus than men. This confirmed that it boosted the girls' immune responses and may have made the vaccine more effective [8]. Genetic factors may also give some guidance on how gender affects infection. Professor Meyaard has found a protein called TLR7, encoded by a gene on the X chromosome, which causes a stronger immune response in women than in men [15]. In the past, we attributed men's short life span to smoking, drinking, high-risk work, high pressure and other factors, but children are not affected by these factors. Our data confirmed that boys are more easily to isolate bacterium compared with girls. Why are boys more likely to be infected? Men and women only differ in that they have one $\mathrm{X}$ chromosome and two $X$ chromosomes $[16,17]$. The result is consistent with the ancient Chinese saying and the view of international scientists [7]: boys are more easily to be infected compared with girls. We fortunately find a new explanation why men live shorter lives than women.

\section{Conclusion}

In our study, we first established a provincial-level pediatric antimicrobial surveillance system (SPARSS) in China, and confirmed that the pathogenic spectrums of children from different development stages are significantly different. Clinical doctors should select reasonable antibiotics according to the laws of the bacterial infection pathogenic spectrum in children of different ages, so as to promote the reasonable and standard application of antimicrobial drugs. In addition, we also find a new explanation for why, generally speaking, men live shorter lives than women. Our work may be helpful to promote the reasonable and standard application of antimicrobial drugs. Clinical doctors should choose the antibiotics reasonably according to the variation pattern of bacterial pathogenic spectrum in children from different age groups.

\section{List Of Abbreviations}

SPARSS Shandong Province Pediatric Antimicrobial Resistance Surveillance System

ATCC American Type Culture Collection

E. coli Escherichia coli

\section{Declarations}

Ethics approval and consent to participate: This study has obtained the ethics approval from the ethics committee of Qilu Children's Hospital of Shandong University. All the parental/guardian consent to participate in the study.

Consent for publication: The authors report no competing interests in this paper. The authors state that they have obtained appropriate institutional review board approval or have followed the principles 
outlined in the Declaration of Helsinki for all animal experimental investigation.

Availability of data and material: The informed data was obtained from all participating hospitals of this study.

Competing interests: None declared.

Funding: This study was supported by Grants from the State Major Infectious Disease Research Program (China Central Government, 2012ZX10004206-007 and 2017zx10103004-007). The founders had no role in the data collection and analysis, decision to publish, or preparation of the manuscript.

Author's Contribution: SFW carried out the design and drafting of the manuscript. Other authors help to collect the data. YKZ and ZTG designed the study, and participated in drafting the article. All authors read and approved the final manuscript.

Acknowledgement: Thanks for all hospitals of Shandong Province Pediatric Antimicrobial Resistance Surveillance System.

\section{References}

[1] Liu YY, Wang Y, Walsh TR, Yi LX, Zhang R, Spencer J, et al. Emergence of Plasmid-mediated Colistin Resistance Mechanism MCR-1 in Animals and Human Beings in China: a Microbiological and Molecular Biological Study. The Lancet Infectious Diseases. 2016;16:161-8.

[2] Vincent JL. Antibiotic Administration in the Ambulance? The Lancet Respiratory Medicine. 2018;6:5-6.

[3] Chandrasekhar D, Dollychan A, Roy BM, Cholamughath S, Parambil JC. Prevalence and Antibiotic Utilization Pattern of Uropathogens Causing Community-acquired Urinary Tract Infection in Kerala, India. Journal of Basic and Clinical Physiology and Pharmacology. 2018;29:671-7.

[4] Lu Y, Wang S, Zhang L, Xu C, Bian C, Wang Z, et al. Epidemiology of Human Respiratory Viruses in Children with Acute Respiratory Tract Infections in Jinan, China. Clinical \& Developmental Immunology. 2013;2013:210490.

[5] Kalenahalli KJ, Kumar NA, Chowdary KV, Sumana MS. Fatal Swine Influenza A H1 N1 and Mycoplasma Pneumoniae Coinfection in a Child. Tuberkuloz ve Toraks. 2016;64:246-9.

[6] Opatowski L, Baguelin M, Eggo RM. Influenza Interaction with Cocirculating Pathogens and Its Impact on Surveillance, Pathogenesis, and Epidemic Profile: A Key Role for Mathematical Modelling. PLoS Pathogens. 2018;14:e1006770.

[7] Reardon S. Infections Reveal Inequality Between the Sexes. Nature. 2016;534:447. 
[8] Jensen KJ, Ndure J, Plebanski M, Flanagan KL. Heterologous and Sex Differential Effects of Administering Vitamin A Supplementation with Vaccines. Transactions of the Royal Society of Tropical Medicine and Hygiene. 2015;109:36-45.

[9] Mulu W, Yizengaw E, Alemu M, Mekonnen D, Hailu D, Ketemaw K, et al. Pharyngeal Colonization and Drug Resistance Profiles of Morraxella catarrrhalis, Streptococcus pneumoniae, Staphylococcus aureus, and Haemophilus influenzae among HIV Infected Children Attending ART Clinic of Felegehiwot Referral Hospital, Ethiopia. PloS one. 2018;13:e0196722.

[10] Benetti G, Cavaliere E, Brescia R, Salassi S, Ferrando R, Vantomme A, et al. Tailored Ag-Cu-Mg Multielemental Nanoparticles for Wide-spectrum Antibacterial Coating. Nanoscale. 2019;11:1626-35.

[11] Bedford T, Riley S, Barr IG, Broor S, Chadha M, Cox NJ, et al. Global Circulation Patterns of Seasonal Influenza Viruses Vary with Antigenic Drift. Nature. 2015;523:217-20.

[12] Rynda-Apple A, Robinson KM, Alcorn JF. Influenza and Bacterial Superinfection: Illuminating the Immunologic Mechanisms of Disease. Infection and Immunity. 2015;83:3764-70.

[13] Klein EY, Monteforte B, Gupta A, Jiang W, May L, Hsieh YH, et al. The Frequency of Influenza and Bacterial Coinfection: a Systematic Review and Meta-analysis. Influenza and Other Respiratory Viruses. 2016;10:394-403.

[14] Lin X, Hull CM, Heitman J. Sexual Reproduction Between Partners of the Same Mating Type in Cryptococcus Neoformans. Nature. 2005;434:1017-21.

[15] Karnam G, Rygiel TP, Raaben M, Grinwis GC, Coenjaerts FE, Ressing ME, et al. CD200 Receptor Controls Sex-specific TLR7 Responses to Viral Infection. PLoS Pathogens. 2012;8:e1002710.

[16] Sanchis-Segura C, Becker JB. Why We Should Consider Sex (and Study Sex Differences) in Addiction Research. Addiction Biology. 2016;21:995-1006.

[17] Austad SN. Why Women Live Longer Than Men: Sex Differences in Longevity. Gender Medicine. 2006;3:79-92.

\section{Tables}

Due to technical limitations, all Tables are only available as a download in the supplemental files section.

\section{Figures}




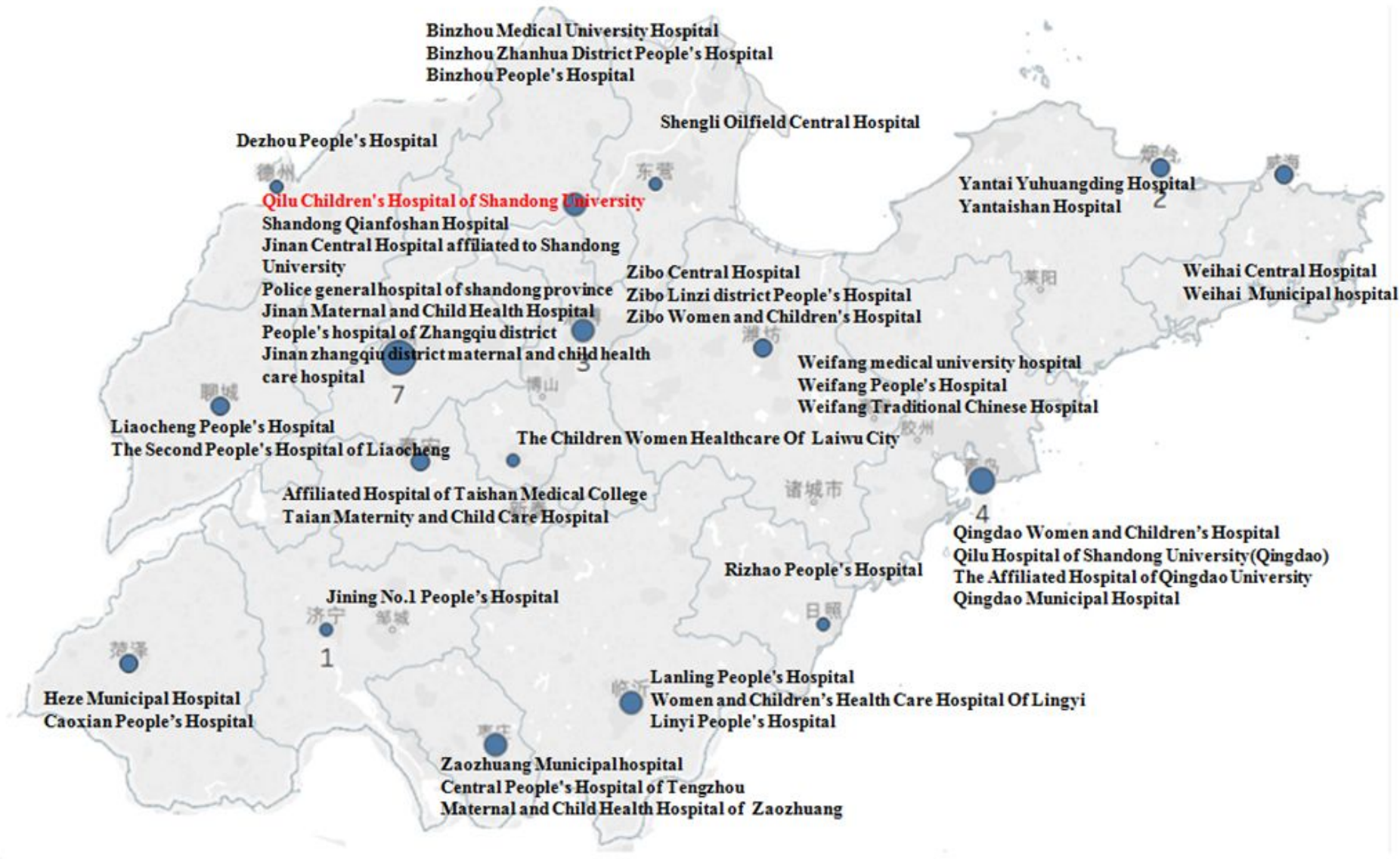

\section{Figure 1}

The member units' geographical distribution of Shandong Province Pediatric Antimicrobial Resistance Surveillance System network (SPARSS net) 


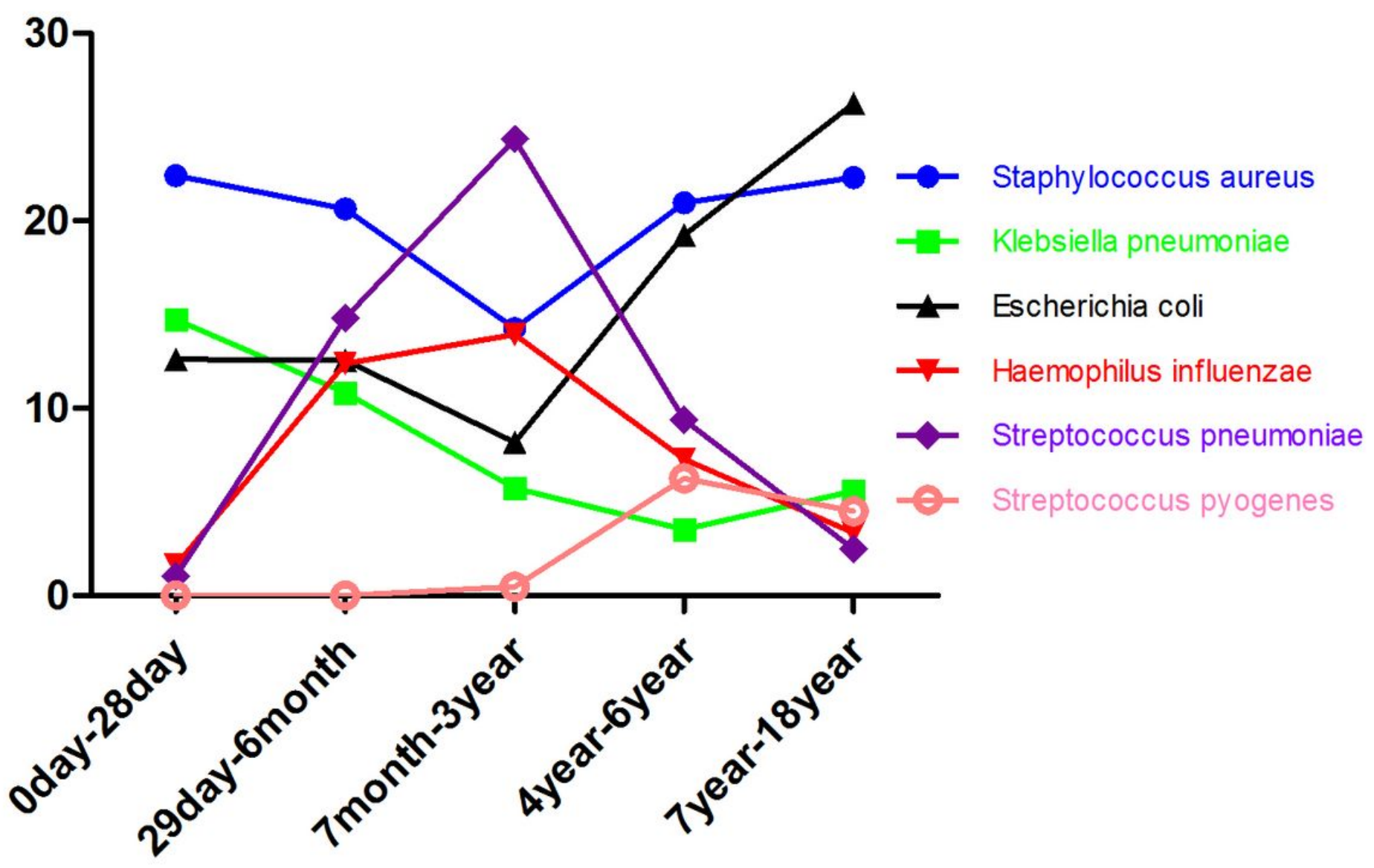

Figure 2

The change trend analysis of main pathogen spectrum composition percentage in children of different age. 


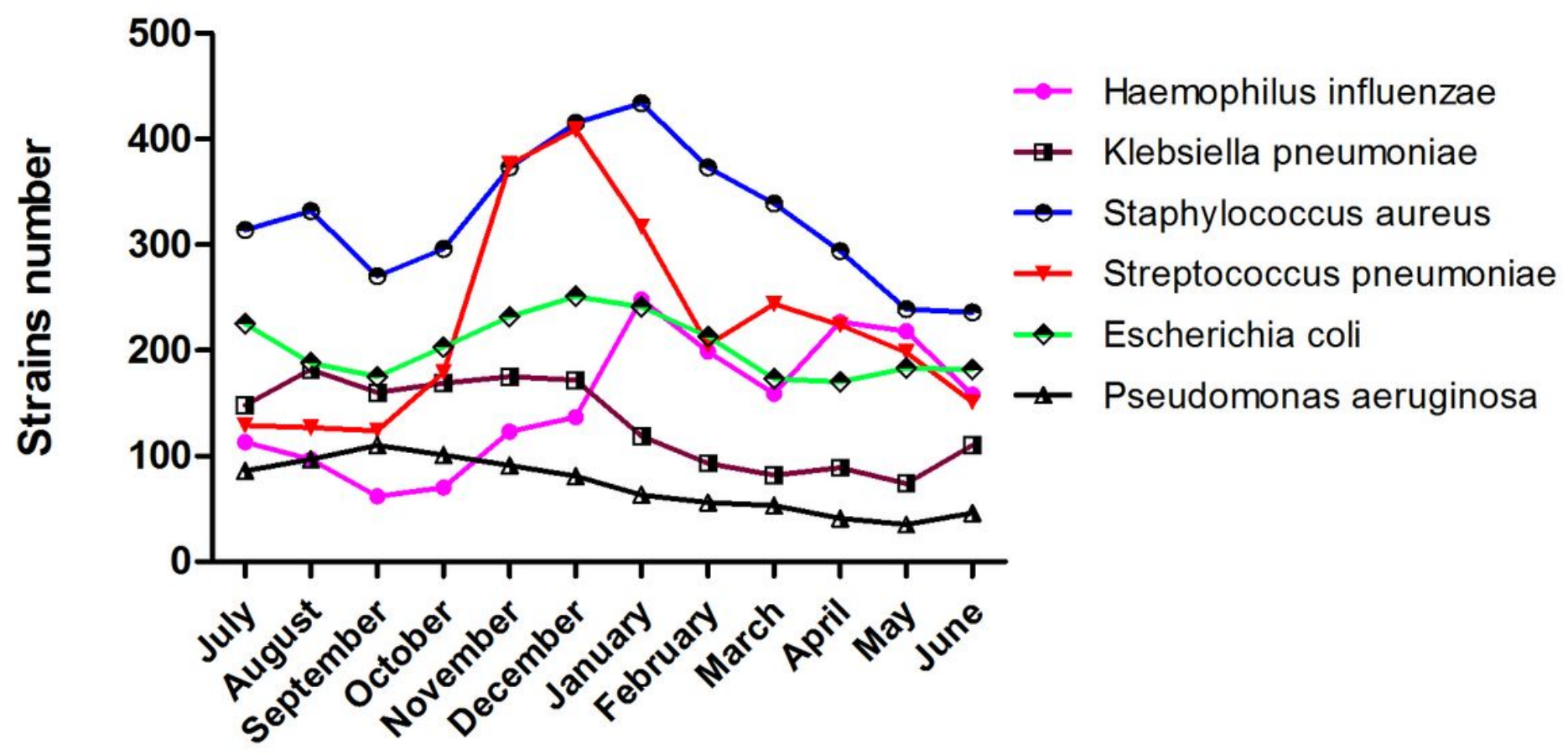

Figure 3

The main bacteria separation for monthly variation 


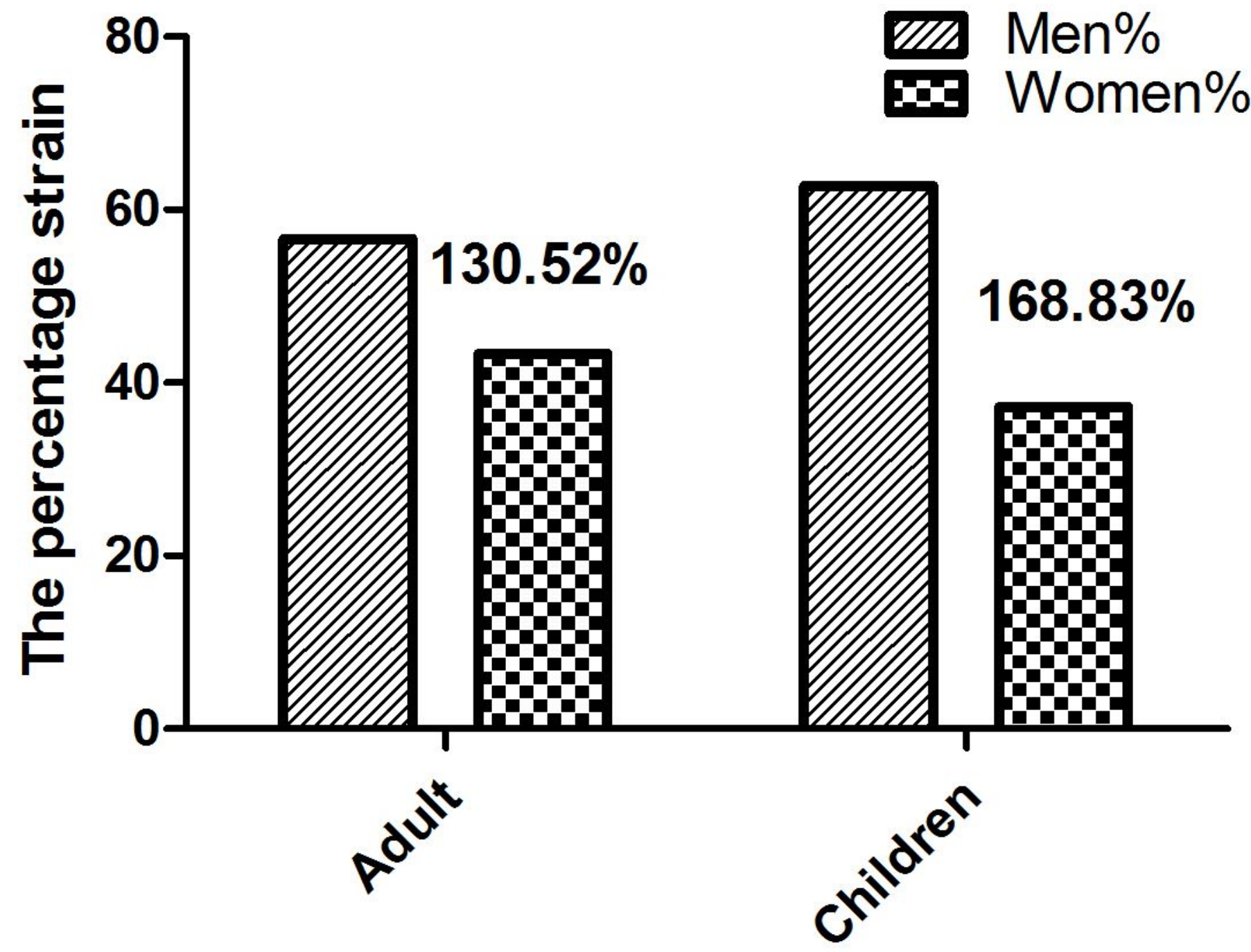

Figure 4

The ratio of strains number separated from different gender for men and women between children and adults

\section{Supplementary Files}

This is a list of supplementary files associated with this preprint. Click to download.

- Table1thecomparisonofpathogenspectrum.jpg

- Table2.Thecomparisonofmainpathogenicspectrum.jpg

- Table3.Theratioofdifferentgender.jpg 